\title{
Complete fabrication of target experimental chamber and implement initial target diagnostics to be used for the first target experiments in NDCX-1
}

F.M. Bieniosek, M.R. Dickinson, E. Henestroza, T. Katayanagi, J.Y. Jung, C.W. Lee, M. Leitner, P. Ni, P. Roy, P. Seidl, W. Waldron, - LBNL D. Welch - Voss Scientific

Accelerator Fusion Research Division

Ernest Orlando Lawrence Berkeley National Laboratory

University of California

Berkeley, California 94720

June 2008

This work was supported by the Director, Office of Science, Office of Fusion Energy Sciences, of the U.S. Department of Energy under Contract No. DE-AC02-05CH11231. 


\title{
HEAVY ION FUSION SCIENCE VIRTUAL NATIONAL LABORATORY
}

\author{
$3^{\text {RD }}$ QUARTER 2008 \\ MILESTONE REPORT
}

Complete fabrication of target experimental chamber and implement initial target diagnostics to be used ror the first target experiments in HDCX-1.

F.M. Bieniosek, M.R. Dickinson, E. Henestroza, T. Katayanagi, J.Y. Jung, C.W. Lee, M. Leitner, P. Ni, P. Roy, P. Seidl, W. Waldron, D. Welch for the HIFS-VNL

June 12, 2008 


\section{SUMMARY}

This milestone has been met. The Heavy Ion Fusion Science Virtual National Laboratory (HIFS-VNL) has completed the fabrication of a new experimental target chamber facility for future Warm Dense Matter (WDM) experiments, and implemented initial target diagnostics to be used for the first target experiments in NDCX-1. The target chamber has been installed on the NDCX-I beamline. This achievement provides to the HIFS-VNL unique and state-of-the-art experimental capabilities in preparation for the planned target heating experiments using intense heavy ion beams. 


\section{INTRODUCTION}

The US heavy ion fusion science program is developing techniques for heating ion-beam-driven warm dense matter (WDM) targets [1-4]. Intense ion beams have several attractive features as a technique for generating WDM. These features include:

-Precise control of local beam energy deposition $\mathrm{dE} / \mathrm{dx}$, nearly uniform throughout a given volume, and not strongly affected by target temperature, -Large sample sizes (about 1 micron thick by $1 \mathrm{~mm}$ diameter),

-The ability to heat any target material, for example, foams, powders, conductors, insulators, solid, gas, etc.

Uniformity of target heating and efficiency of beam energy deposition are obtained by heating with the Bragg peak at the center of the target. This approach allows operation with moderate beam energy ( $\sim \mathrm{MeV} / \mathrm{AMU})$. Other scenarios take advantage of two other regions where the $\mathrm{dE} / \mathrm{dx}$ curve for heavy ions is nearly flat: high energy approaching $1 \mathrm{GeV} / \mathrm{AMU}$ (as at GSI), and low energy $\sim 10 \mathrm{keV} / \mathrm{AMU}$. The range of the low and moderate energy beams planned for the HIFS-VNL experiments is about 1 micron in solid matter targets, which can be lengthened by using porous targets at reduced density. Because of the short range, it is necessary to compress beam pulses to roughly $1 \mathrm{~ns}$ to be consistent with the hydrodynamic expansion time of the target. The range can be extended by heating low-density porous targets, for example density in the range of $1-10 \%$ of solid density, extends ion beam range and hydrodynamic expansion time by factors of 10-100. Initial experiments will be at low beam velocity, below the Bragg peak using the existing NDCX-1 accelerator (0.3-0.4 $\mathrm{MeV} \mathrm{K}^{+}$). Intense ion beam currents must be focused to a spot size less than $1 \mathrm{~mm}$ in order to achieve sufficient heating power on target for these low kinetic energy ions. This is only made possible by the neutralized drift compression technique recently developed in the HIFS-VNL. The ion beam will undergo combined longitudinal and transverse neutralized drift compression to provide a hot spot on the target with a beam spot size $\leq 1 \mathrm{~mm}$, and pulse length about 1-2 $\mathrm{ns}$.

Figure 1 illustrates schematically the required components for such warm dense matter heating experiments.

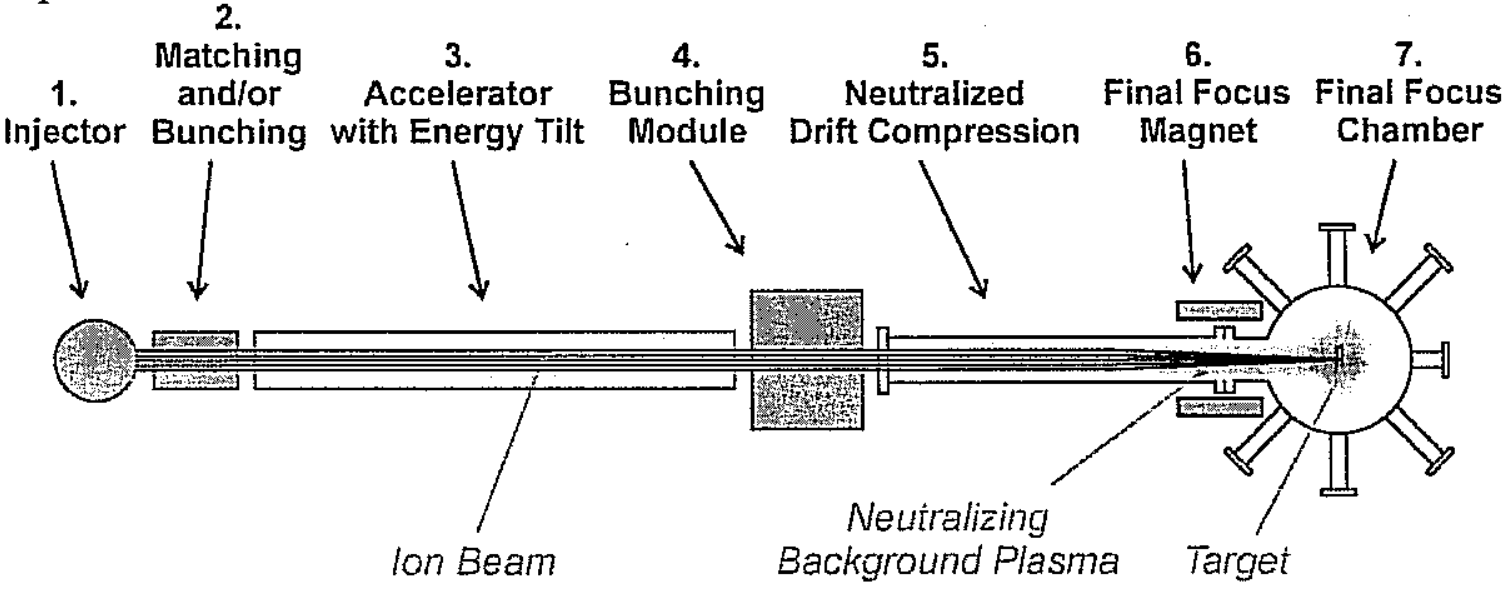

Figure 1: Experimental setup for heavy-ion heated WDM targets. 
This approach has significant consequences for the experimental target and target chamber setup. Design of the experimental target chamber was reported in the $4^{\text {th }}$ Quarter 2007 milestone report. The target chamber as built is designed to provide:

1. sufficiently dense $\left(10^{12}\right.$ to $\left.10^{14} \mathrm{~cm}^{-2}\right)$ plasma injection to neutralize the space charge of the incoming ion beam,

2. a strong ( 8 Tesla) final focus solenoid for radial ion beam compression,

3. vacuum pumping,

4. retractable ion beam diagnostics,

5. a retractable target holder with in-situ alignment capabilities,

6. fast optical target diagnostics with access to the front, side and back of the target foil,

7. accurate (micron range) alignment capabilities

We have developed a WDM target chamber and a suite of target diagnostics including a high speed multi-channel optical pyrometer, optical streak camera, VISAR, and high-speed gated cameras. The target chamber and diagnostics will be installed downstream of the induction bunching module on NDCX-1 (Fig. 2). We are investigating the properties of a gold cone for focusing a moderate energy ion beam on target. The cone has been modeled with the TRIM code and prototypes tested on the $300-\mathrm{keV}$ potassium NDCX-1 beam.

Initial WDM targets will provide the opportunity to gain experience with diagnostics for WDM targets. The targets will be approximately a range thick, for example 350-nm Al and 150-nm Au. Simulations of target heating using HYDRA indicate heating to approximately $0.2 \mathrm{eV}$, depending on beam final focus parameters achieved in initial experiments. We have begun a series of planned experiments in warm dense matter. These experiments include a 2006 porous target experiment at GSI to compare response of solid and porous targets, and planned or possible experiments at NDCX-1 or NDCX-2 such as

-initial beam-driven target experiments, expected in mid-2008

-low density porous targets

-high electron affinity (e.g. halogen) target study

-two-phase liquid/vapor targets to study fragmentation and droplet formation

-beam - shock wave coupling in cryogenic targets

-EOS studies

The following sections describe in detail the subcomponents of the new HIFS-VNL target chamber facility and diagnostics.

\section{TARGET CHAMBER COMPONENTS}

Figure 2 displays a schematic view of the new target chamber facility with the major components highlighted. The longitudinally compressing ion beam enters from the left and gets focused to less than $1 \mathrm{~mm}$ diameter by a strong, pulsed solenoid. Because of the low energy of the incoming 
ion beam the focal length of the final focus solenoid is short $(-40 \mathrm{~cm})$. This puts a major constraint on the target chamber design since each sub-component has to be tightly packed in space.

The target chamber is a precisely machined octagon made of 304 stainless steel as shown in figure 2. The octagonal shape allows the precise arrangement of different diagnostics around the side of the target which is located in the exact center of the chamber. In addition, the front entrance flange as well as the exit flange provide 4 vacuum ports each which allow to look straight through the target plane.

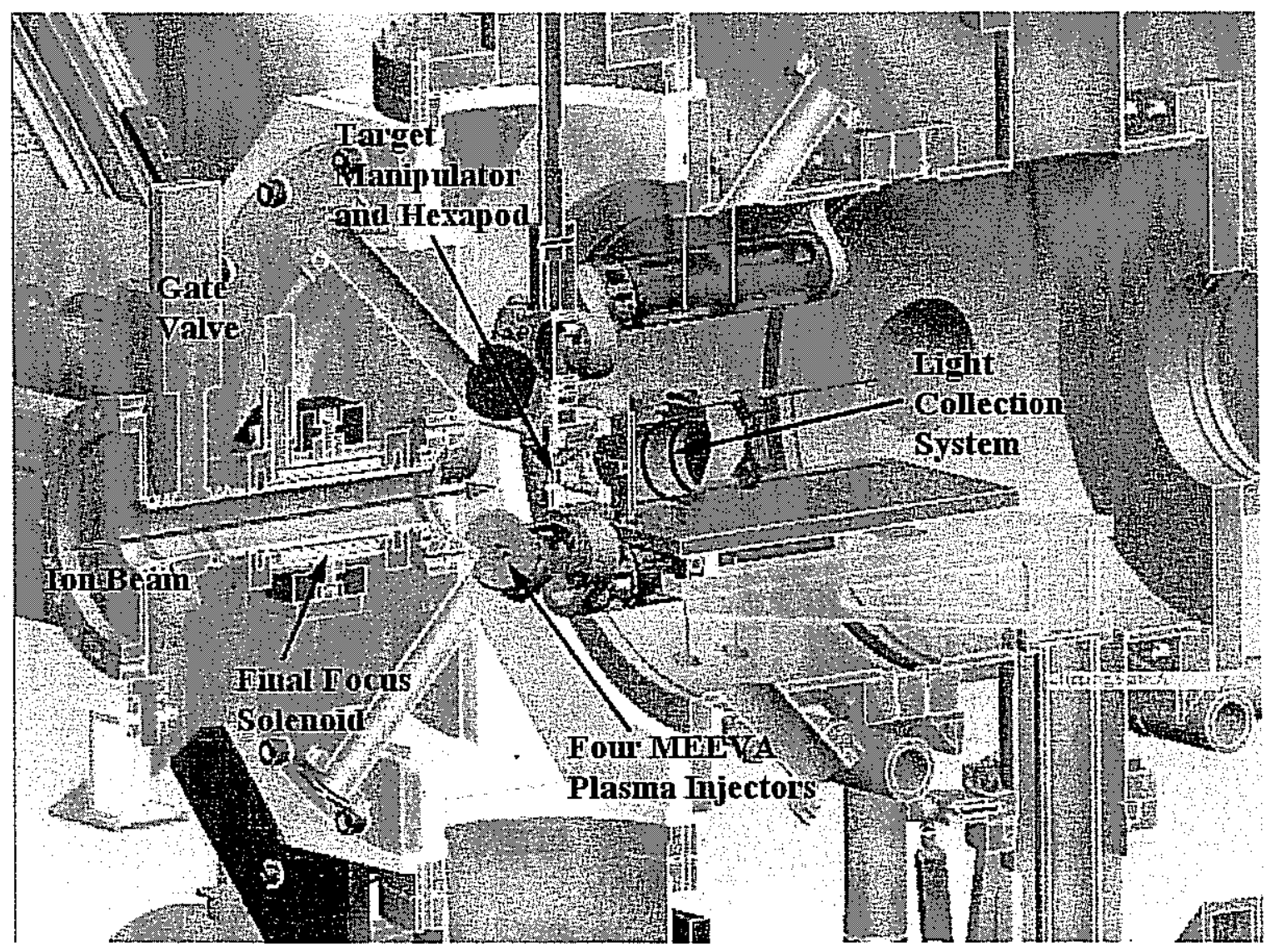

Figure 2: 3D CAD view of the target chamber. The target foil is mounted in the exact center of the chamber with the ports aiming at the target location.

A small vacuum gate valve in front of the final focus solenoid allows decoupling the target chamber vacuum from the accelerator beamline in order to vent the target chamber for target changes, maintenance, or swapping of diagnostics. The bottom chamber ports are reserved for two vacuum pumps, a $1000 \mathrm{l} / \mathrm{sec}$ cryopump with gate valve and a $500 \mathrm{l} / \mathrm{sec}$ turbo pump, an ion gauge and a vent port. 
Design of the 8-Tesla final focus solenoid magnet is described in detail in Ref. 5. The solenoid has operated reliably for plasma injection studies.

\subsection{PLASMA INJECTION}

Figure 3 shows the location of the four filtered cathodic arc plasma source (FCAPS) plasma guns used to inject plasma into the final focus region right in front of the target.

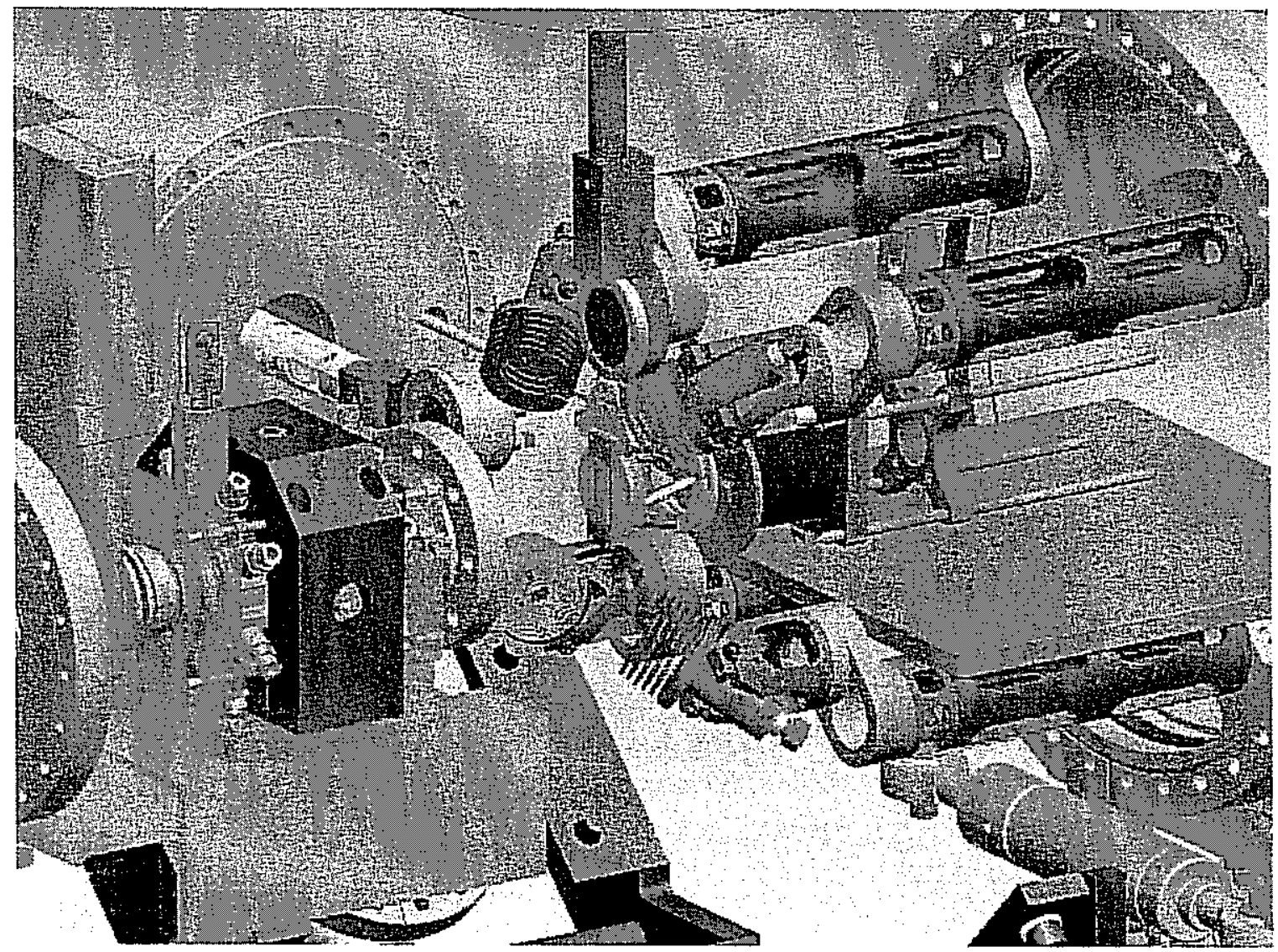

Figure 3: Four FCAPS plasma guns (aimed towards the front of the target) inject plasma for ion beam space charge neutralization.

Significant time has been expended to minimize the size of the target holder and ion beam diagnostics footprint in order to maximize the plasma flow to the target area. The plasma flow is slowed down once entering the high field region of the final focus solenoid and becomes confined in front of the target. Figure 4 shows a photograph of the plasma flow inside the target chamber. 


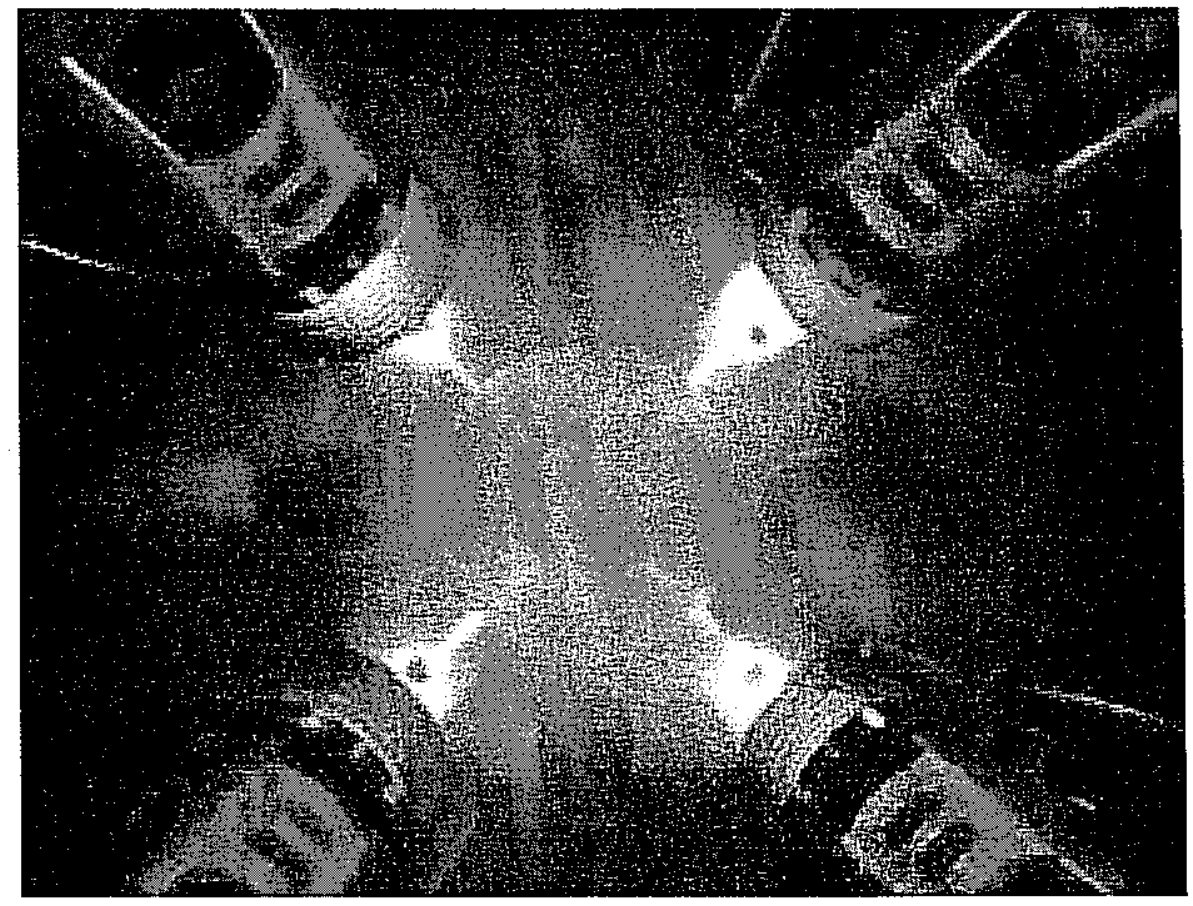

Figure 4: Photograph showing the plasma glow during the firing of the FCAPS plasma injectors.

The distribution of plasma within the target chamber has been measured with a Langmuir probe array. The measured plasma shows that the $8 \mathrm{~T}$ solenoid field increases the plasma density on the beam axis as expected qualitatively. Figure 5 shows surface plots of the measured transverse plasma distribution at the center of the final focus solenoid without (Fig.5a) and with (Fig.5b) the magnetic field. Using a measured velocity of $5 \times 10^{3} \mathrm{~m} / \mathrm{sec}$, the maximum density at this location is $1.6 \times 10^{13} \mathrm{~cm}^{-3}$ for the measured plasma current of $53 \mathrm{~mA}$ for $\mathrm{Al}^{2+}$. The area of a collector is $2.01 \mathrm{~mm}^{2}$. The probe array (Fig.5c) consists of 37 collectors. Each collector is operated in the ion saturation regime ( -70 volts). 
a)

Furfase plat, THA., B*0

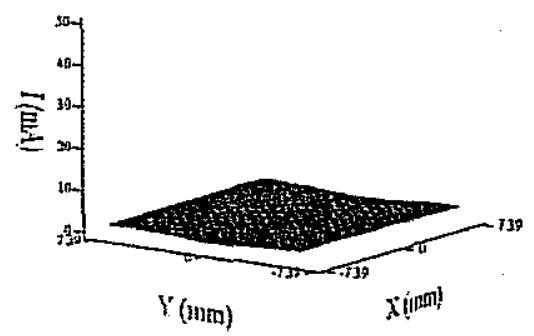

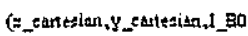

c)

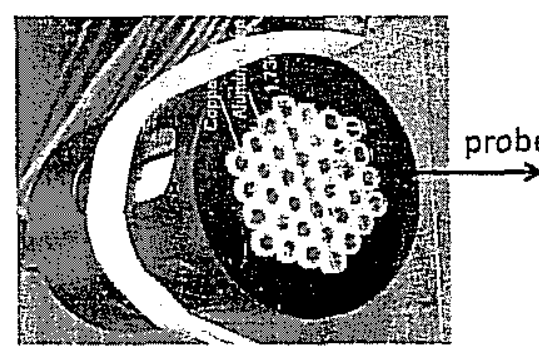

b) Suface fioh TIOA, EugT

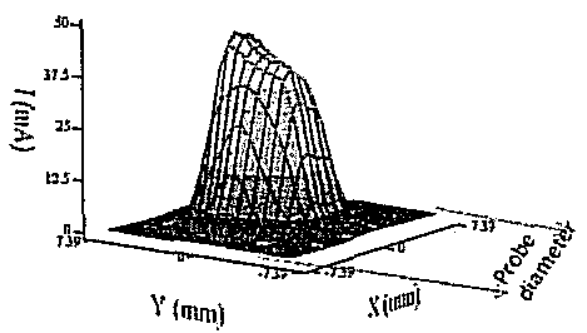

d)

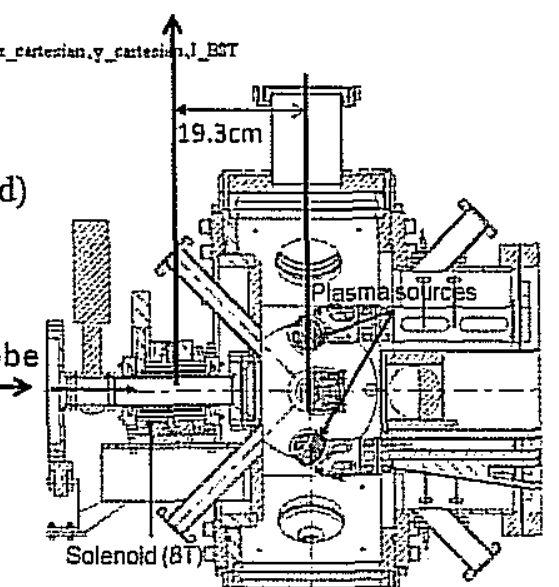

Figure 5. Measured plasma distribution at the center of the final focus solenoid $(19.3 \mathrm{~cm}$ from the target plane) (a) without and (b) with the magnetic field, (c) plasma probe array and (d) target chamber. Four (FCAPS) operated with a discharge current of $770 \mathrm{~A}$.

Recent simulation results obtained using the LSP code of the plasma loading into the final focus solenoid and target region are shown in Fig 6. A plasma density comparable to or greater than the peak beam density is required for good charge neutralization and a small spot on target. These initial 3D simulations track injected plasma ion trajectories in the applied magnetic fields (ignoring plasma self fields). We compare the simulated plasma density, in the current experimental setup, using magnetic fields produced by a static solution of the applied fields (from both the $8 \mathrm{~T}$ final focus solenoid and the 1-kG FCAPS coils) with those produced from a time-dependent diffusive calculation incorporating the effects of eddy currents in nearby structures (supplied by J-Y Jung using the Ansys code). As can be seen in Fig. 6, the "static B" map yields an annular plasma density within the solenoid while the "diffusive $B$ " map produces a more uniform density. Due to the less-extended lines of force, the diffusive B map also permits more plasma in the vicinity of the target downstream of the final focus solenoid. In both simulations, the plasma is cut off beyond a 1-cm radius in the solenoid. The diffusive B map results are more consistent with the experimental measurements. Electromagnetic LSP simulations, and Warp simulations, are underway to confirm these results. 

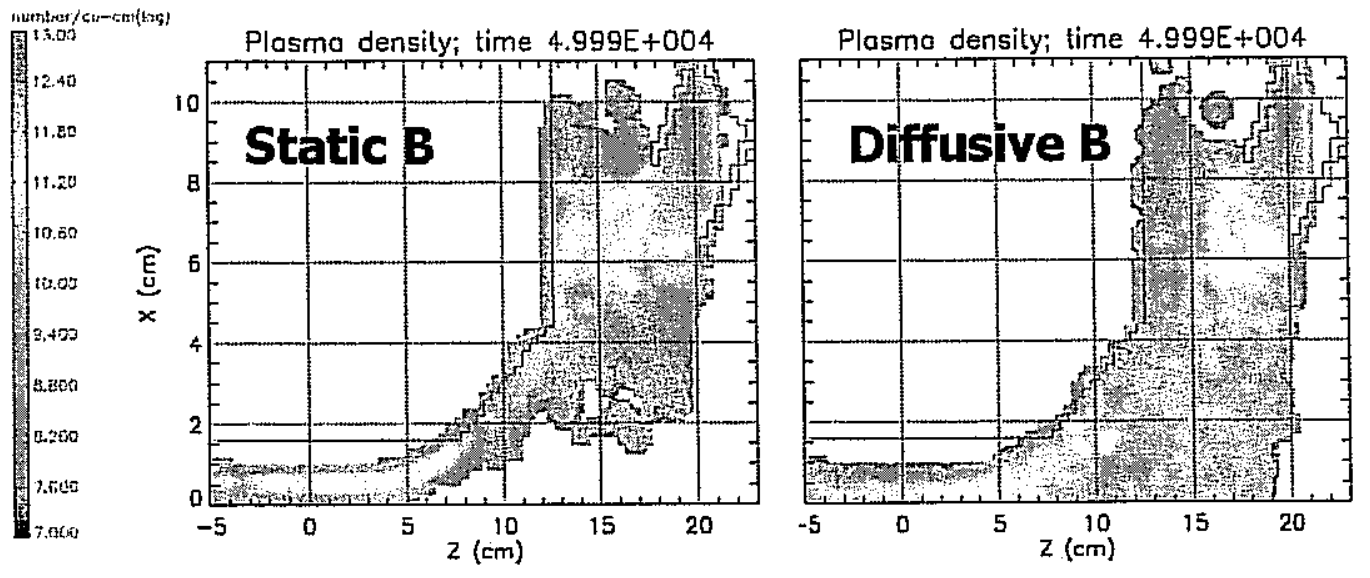

Figure 6. The LSP-calculated plasma density is shown for the static field (left) and diffusive field (right) simulation after 50,000 ns. Plasma is injected through the FCAPS source at the upper right towards the solenoid centered at $\mathrm{z}=0$. 


\subsection{ION BEAM DLAGNOSTICS AND TARGET MANIPULATOR}

As shown in Fig. 7, two target chamber ports are reserved for ion beam diagnostics as well as target manipulation. A fast Faraday cup is mounted on the horizontal vacuum motionfeedthrough. It is a modified design, with better electrical noise isolation against the nearby signals generated by the cathodic-arc plasma sources (the housing is entirely constructed of metal parts) and the design is more modular to facilitate replacing the hole plates with plates of different geometric transparency. The transverse dimensions of both the scintillator and Faraday cup were reduced to fit in the smaller space between the new FCAPS sources (see Fig. 3 and 4). A scintillator plate and the target holder are mounted on a vertical $x-y$ motion stage allowing movement in and out of the target chamber as well as movement along the beam axis.

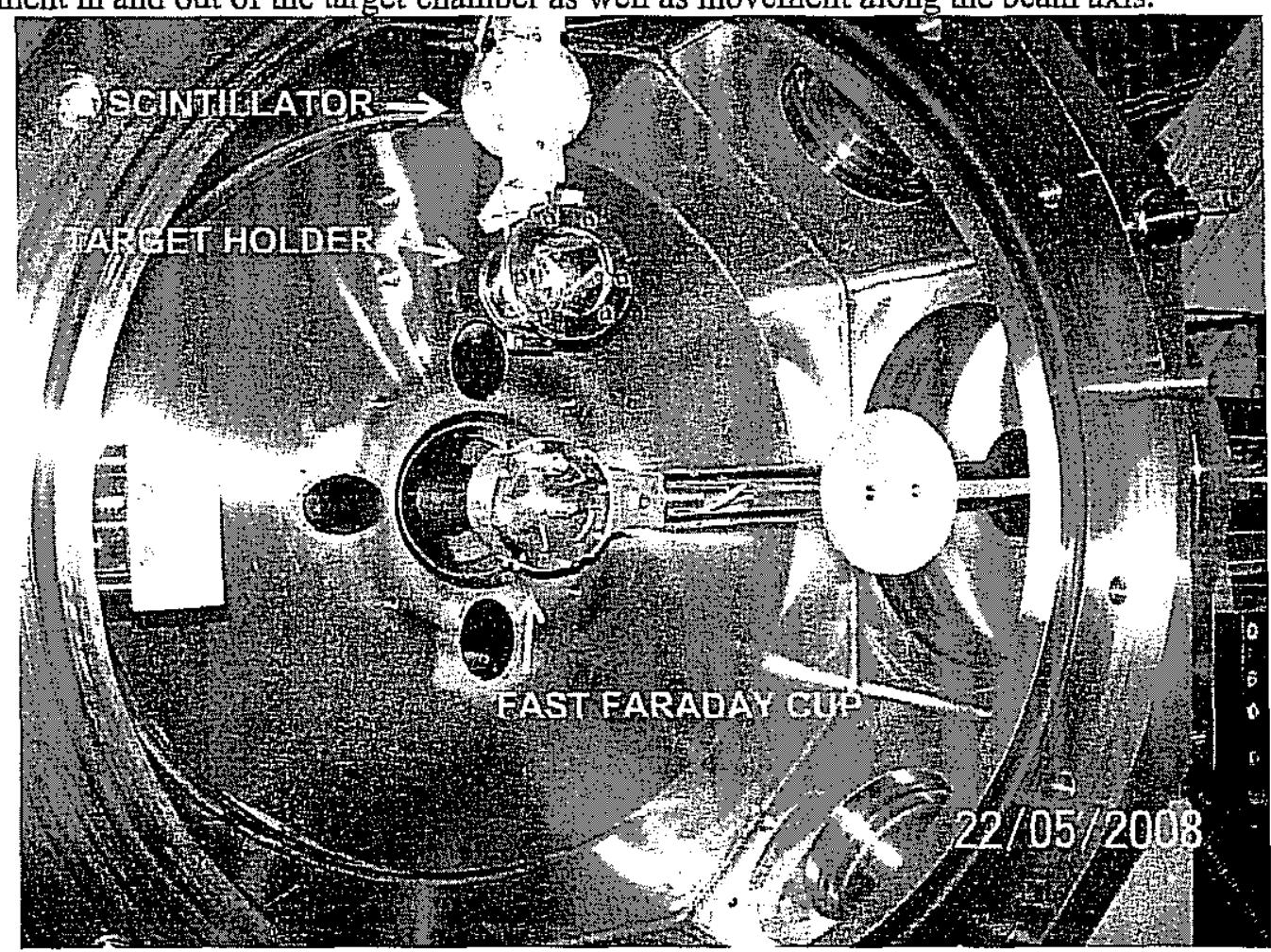

Figure 7: Photograph taken from the rear of the target chamber showing the two motion feedthroughs with the scintillator, fast faraday cup, and the target holder.

Since the target foil is less than a micron thick the correct positioning of the target is particularly critical. It must be aligned to both the incoming ion beam and the whole array of target diagnostics devices. We designed a manipulator with the future development of a target robot system in mind. For a WDM user facility the goal is to have a robotic target handling system which can automatically choose and position from 20 to 50 preloaded target assemblies without breaking vacuum.

The target has to be able to be aligned in the $\mathrm{x}-, \mathrm{y}-$, and $\mathrm{z}$-directions, as well as in roll, pitch, and yaw with respect to the ion beam axis. The most compact device for this type of positioning is a 
"parallel kinematic manipulator", or hexapod. As a future development goal we are envisioning a robot arm, which grabs a preloaded target from a vacuum load lock system and moves it into the target area. For the in-situ target alignment a miniature hexapod will sit on the end of that robot arm.

Our current target manipulator consists of such an arm with a manually adjustable hexapod on top as shown in Fig. 7. A long vacuum translational feedthrough allows retracting the target from the target chamber center in order to be able to insert ion beam diagnostics. Once the ion beam has been tuned and diagnosed for a particular set of target experiments, the target manipulator moves the target holder into the center of the target chamber.

The miniature hexapod and the beam diagnostics must be compact to minimize shadowing of the injected neutralizing plasma flow. At the same time it has to allow visual access to the side, back, and front of the target foil. No such hexapod is commercially available. We have decided to develop our own system by first implementing a manually adjustable hexapod. We will assess the usefulness our chosen design strategy and improve it for an eventually fully automated robot system. Currently, in order to replace target foil without requiring a time-consuming cooling and reheating of the ion source, the target chamber can be isolated from the upstream beamline vacuum with a gate valve and vented to air. As a next step we plan to incorporate a vacuum load lock system to facilitate a quicker target replacement time. 


\subsection{INITLAL TARGETS}

The target is mounted in the center of the octagonal shaped target chamber and has diagnostic access from the front, rear and side. Initial experiments will provide an opportunity to gain experience with delivering the beam at final focus, as well as with diagnostics on beam-heated WDM targets.

Initial experiments will focus on characterizing the NDCX-1 beam at final focus. In these studies the beam will be imaged from the rear on a solid scintillator using a PIMAX camera, or a phototube, or the WDM target diagnostic fiber optic system described below. Initially we plan to use the same alumina scintillators that we have used extensively for beam characterization in our experimental. work. Alumina is known to be relatively robust to beam damage, and previous measurements show sufficiently rapid response to follow the beam intensity on target on the nanosecond time scale. If alumina does not survive the intense beam pulses, or if it does not exhibit a linear response at very high intensity, we have some (5-mm diameter) diamond targets that may prove to be more robust than alumina. Further measurements can be made using the gas cloud from a hole plate, as well as gas-based diagnostics based on beam-induced fluorescence on noble gases such as xenon.

After characterizing the beam, a WDM target will be installed at the focal point of the beam. Targets in hand include free-standing foils (350-nm Al, 150-nm Au) and thin films deposited on a sapphire substrate (350-nm Al, 150-nm Au and 150-nm W). These targets have a thickness comparable to the range of the $300-\mathrm{keV} \mathrm{K}^{+}$beam from NDCX-1, so that the energy deposition distribution is nearly uniform. Figure 8 shows some simulations using HYDRA of the temperature distribution in typical targets for an assumed beam energy delivered to the target of 2-A, 2-ns, $1-\mathrm{mm}$ diameter, $350-\mathrm{keV} \mathrm{K}^{+}$beam. This corresponds to an energy density of 0.1 $\mathrm{J} / \mathrm{cm}^{2}$. In this calculation tin (Sn) was used in place of gold in order to be able to use the QEOS equation of state tables. In general the temperature of a target increases as the target atomic mass increases, so that the gold and tungsten targets are expected to reach a higher temperature than indicated here for tin and aluminum. Note that, except for case " $E$ " indicated on Fig. 8, there is no pre-pulse in the beam. The effect of the pre-pulse will be to increase the temperature of the target before the main heating pulse arrives. 


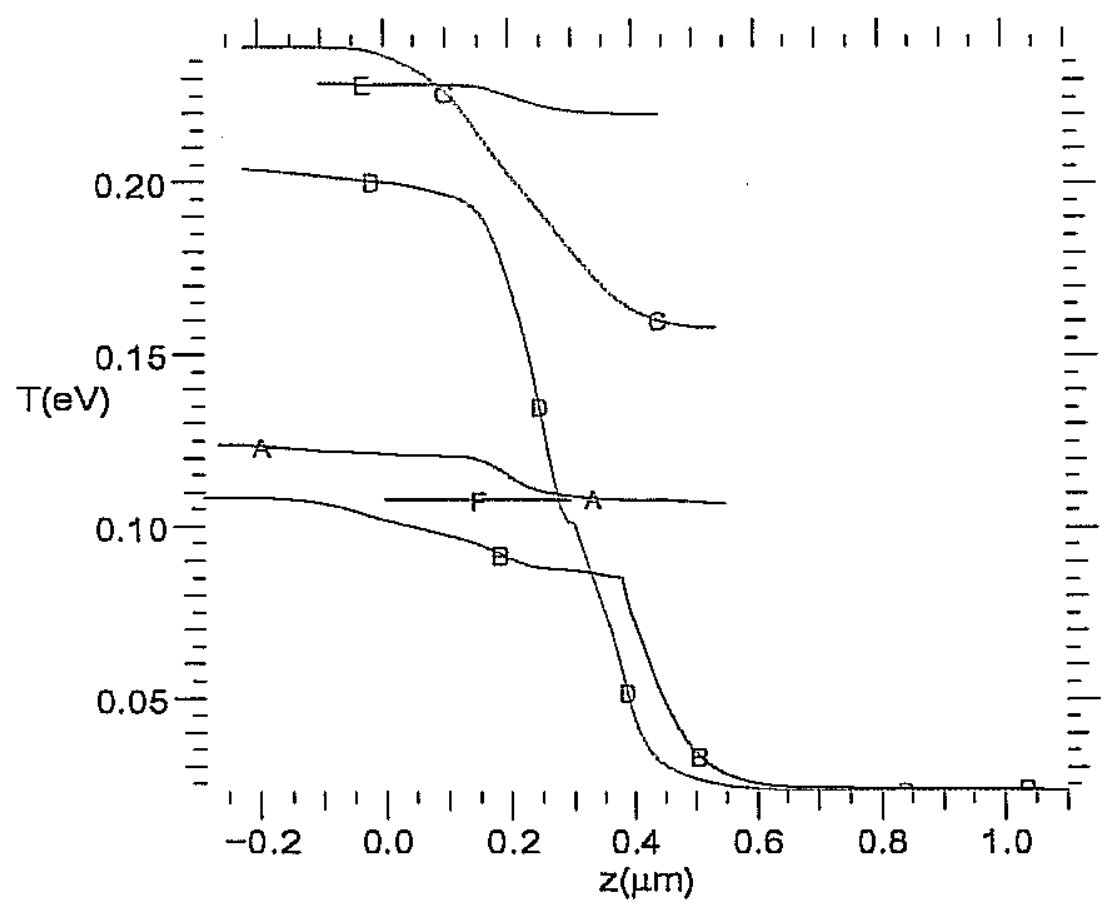

Fig. 8. HYDRA simulations of temperature profile for various target configurations at $t=8 \mathrm{~ns}$. Key: A) Al free standing, B) Al on sapphire, C) Sn free standing, D) Sn on sapphire, E) Sn free standing with $1-\mu$ s prepulse, F) $\mathrm{C}$ free standing. The initial front surface of the target occurs at the position $\mathrm{z}=0$. 


\section{TARGET DIAGNOSTICS}

The primary target diagnostics are a fast pyrometer for temperature measurements, streak camera, spectrometer, VISAR and an optical camera for viewing the target from the rear. The light collection system, which is shown in figure 9, and fiber optics lines and fiber optics feedthroughs. A schematic of the setup of the light collection system is shown in figure 10 .

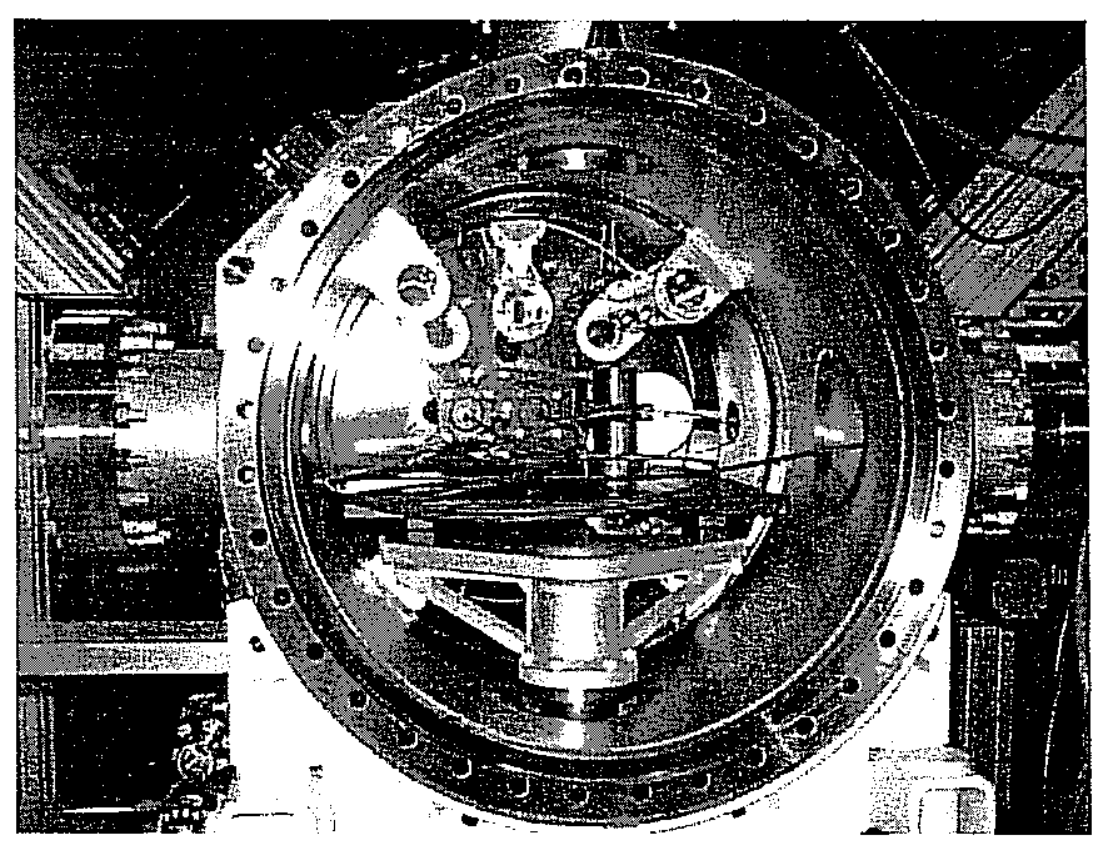

Figure 9: The fiber-coupled light collection system is shown in this image taken from the rear of the target chamber. It is installed in a separate vacuum spool, which can be removed from the main target chamber vessel for servicing. A hexapod, adjustable from outside the vacuum, allows precision alignment of the optical table.

One version of the light collection optics is based on two off-axis parabolic mirrors. Mirrors with large focus and diameter are used in order to capture the spatial angle corresponding to $\mathrm{NA}=22$ (24 degrees) of a conventional multimode fiber. The resulting coupling efficiency is about $50 \%$, possibly limited by coma and sensitivity to misalignment.

An alternative collection optics based on two achromatic lenses (Rayleigh lens system) has been implemented as well. It has a coupling efficiency $\sim 80 \%$ and allows for direct imaging of the target using a pellicle 1 micron beam splitter and an image guide (to help in alignment and finding the beam spot), as shown in Figs. 10 and 11. A drawback of any glass lens system are chromatic aberrations, resulting in wavelength dependence of the probing spot. It has been measured that the probing spot at $1000 \mathrm{~nm}$ is $5 \%$ bigger than at $750 \mathrm{~nm}$. The effect of aberrations with the 400 micron fiber is negligible if the target's temperature is homogenous within a 500 micron. If a smaller fiber (e.g. 100 micron) is used then the optics views the target as an infinite homogenous plane and aberration are not a concern. 
$2 \times$ NIR achromatic coublets, $f=75, d=50.8$

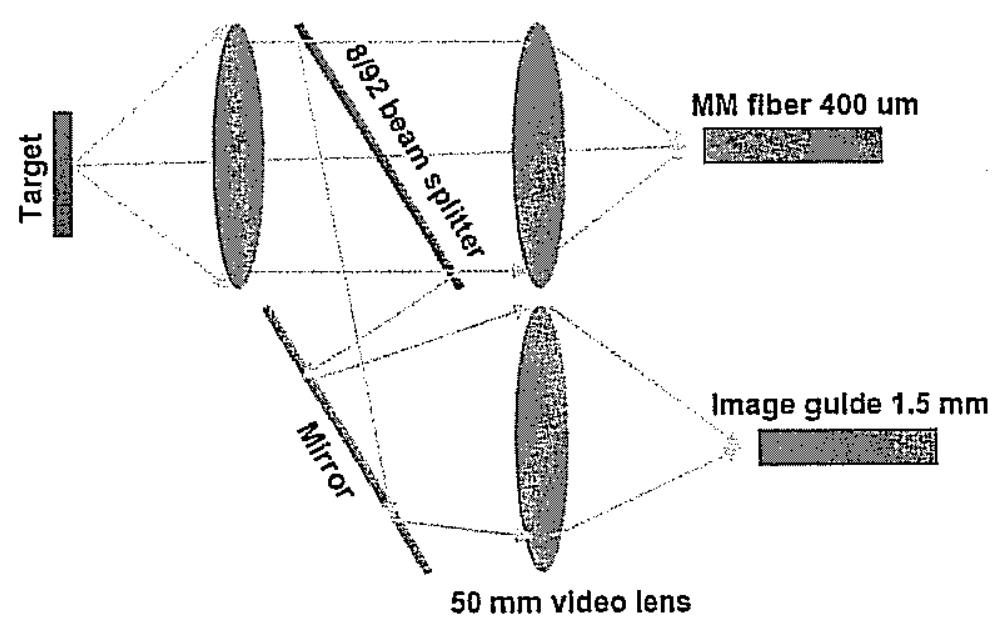

Figure 10. Schematic of optics of light collection system. The $\mathbf{4 0 0}$ micron fiber carries the optical signal to the pyrometer; the image guide provides an image of the beam spot on target to a camera.

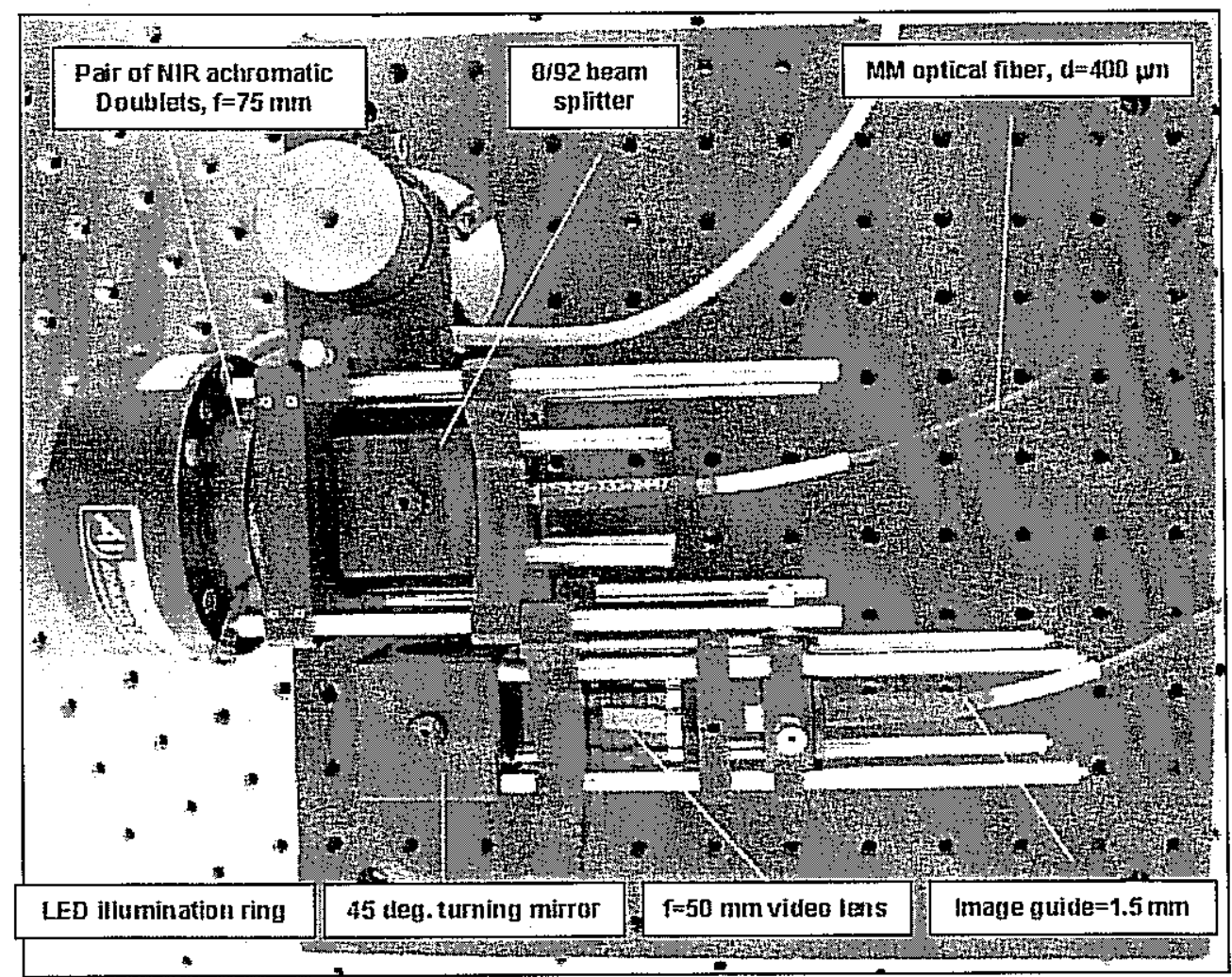

Figure 11. Photograph of light collection system. 


\subsection{Pyrometer}

A pyrometer based on the pyrometer developed at GSI has been developed and commissioned on a laser-based WDM experiment at the ALS. It is ready for the upcoming beam experiments. The main challenge when compared to the GSI pyrometer is the extremely fast (sub-ns) time resolution and higher sensitivity, i.e. ability to measure $\mathrm{T}$ from $1500 \mathrm{~K}$ to $5000 \mathrm{~K}$ (at GSI these values are $5 \mathrm{~ns}$ resolution $\mathrm{T}$ from $2000 \mathrm{~K}$ to $20000 \mathrm{~K}$ ). In order to fulfill these requirement, much higher efficiency spectral resolution, optical beam transport and appropriate type of detectors were investigated.

The main working scheme is shown in Figs. 12 and 13. We use custom broad-band-spectrallyselective beam splitters which have transmission at the level of $90 \%$ (interference filters have $30 \%-70 \%$ ) in order to maintain high sensitivity of the system.

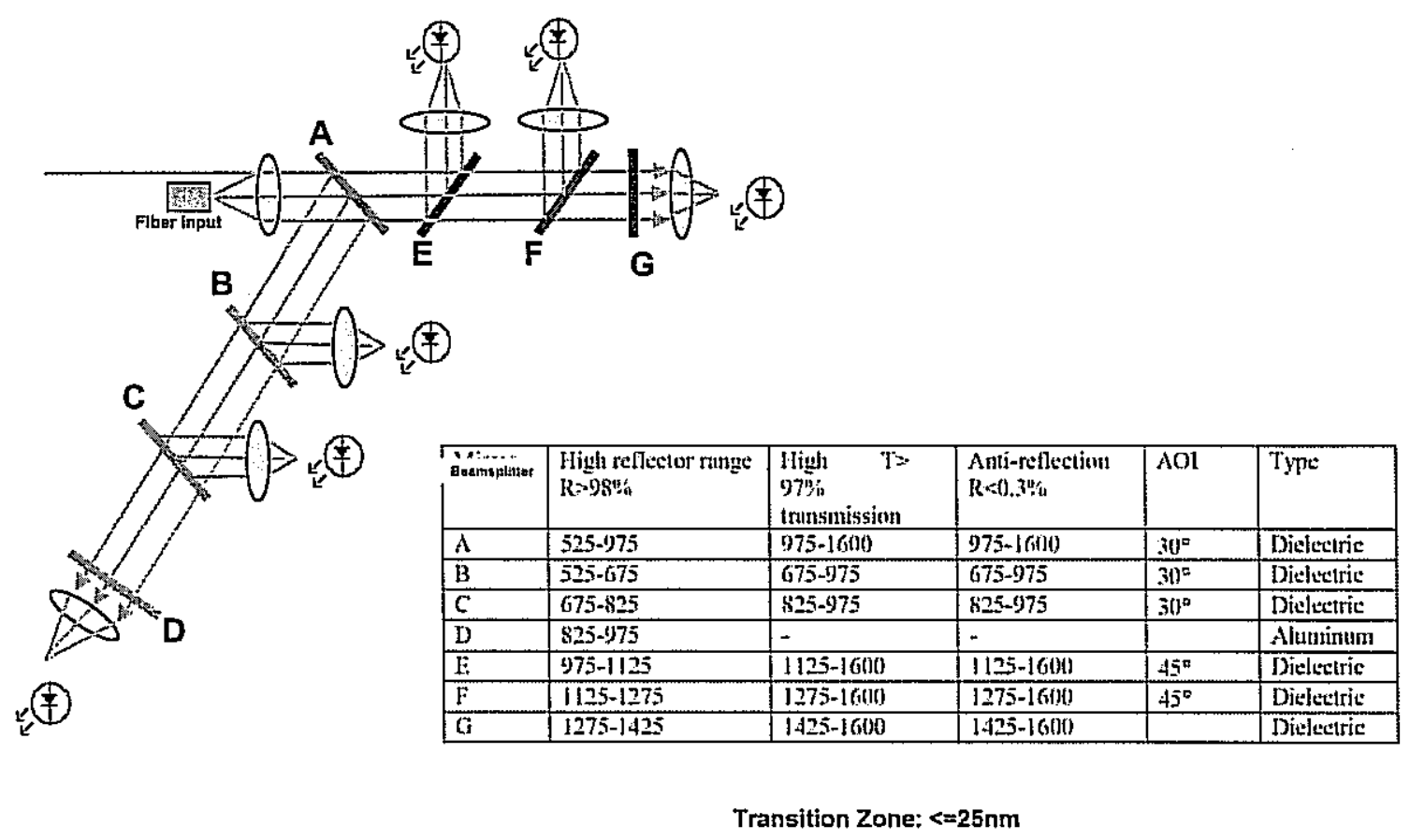

Figure 12. Optical working scheme of pyrometer. The specs of the beam splitter/filters are indicated in the table. 
A photo-detector sensitive to $\sim 1 \mathrm{~mW}$ of light from $400 \mathrm{~nm}$ to $2000 \mathrm{~nm}$ in a sub-ns time range is required. In addition, an extremely broad band and flat amplification gain curve from DC to 4 $\mathrm{GHz}$ is necessary in order to be able to calibrate the pyrometer with DC tungsten filament lamp and at the same time detect temperature behavior features on a sub-nanosecond time scale. Also a relatively big $(0.5 \mathrm{~mm})$ detector size for a simplified alignment is desirable.

We have tested several options, including PIN photo receivers from FEMTO, Newfocus and photomultiplier tubes (PMT). We have chosen the Newfocus due to the flat gain, 75 ps response time and a reasonable price, however these detectors are fiber coupled which is associated with losses in efficiency. Two channels are pictured in Fig. 13. Since this picture was taken we have installed the third channel, and more channels will be added in the future.

Calibration of the pyrometer is carried out with a tungsten ribbon lamp ( $T=2600 \mathrm{~K}$ ), and produces $2 \mathrm{mV}$ voltage at $\mathrm{C}$ channels and $5 \mathrm{mV}$ at $\mathrm{E}$ channel. Noise rms is $0.05 \mathrm{mV}$. Saturation voltage (non-linear regime) is $1.8 \mathrm{~V}$.

Further efficiency improvements with tapered fibers and/or beam expanders are planned.
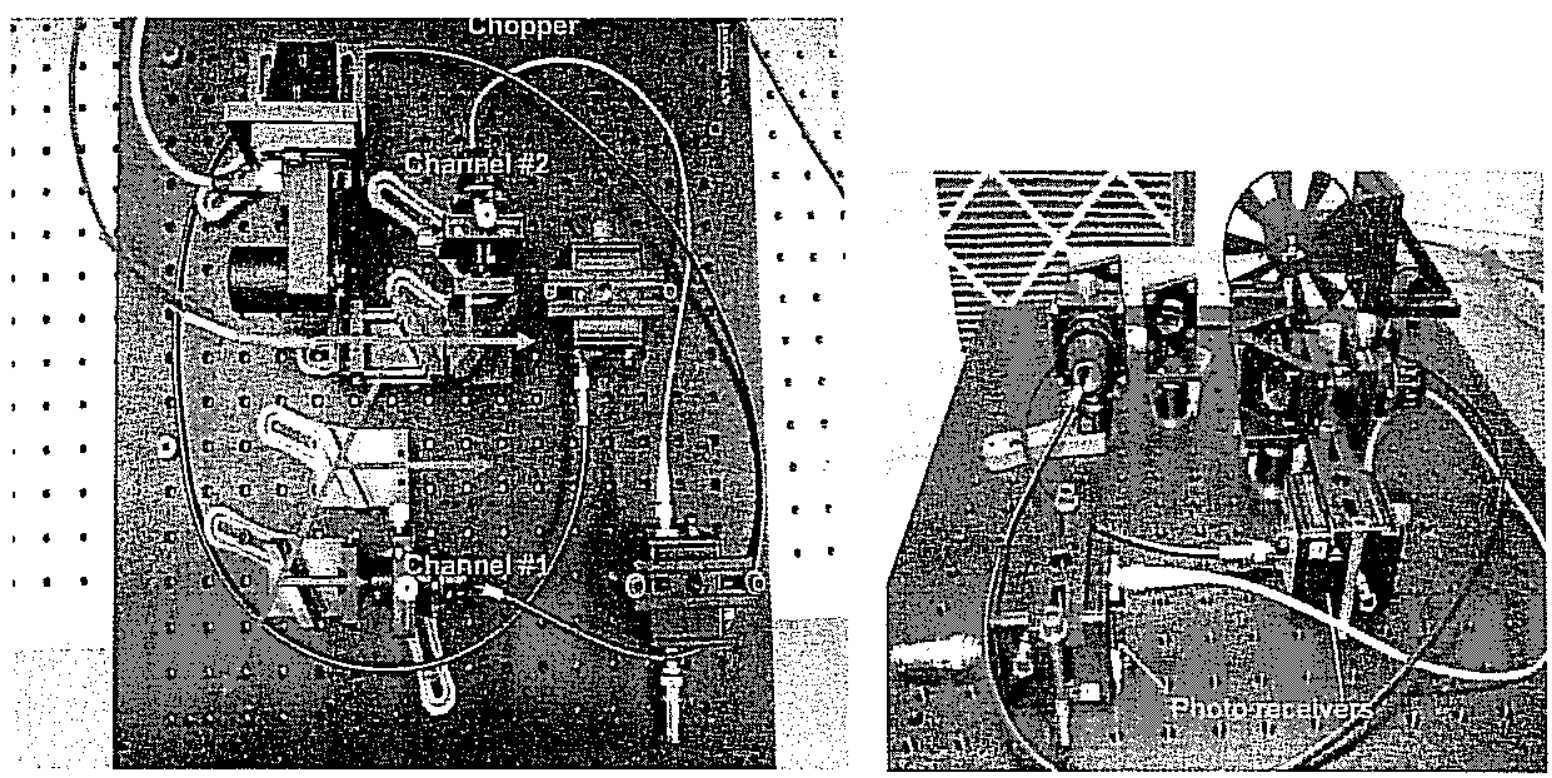

Figure 13. Photographs of the pyrometer as configured with two channels.

The pyrometer has been tested in a laser-driven WDM experiment at one of the beamlines of the Advanced Light Source (ALS) at LBNL. The experimental setup is shown in Fig. 14 and typical results of the target temperature during the experiment is shown in Fig. 15. These results indicate that the pyrometer as fielded at ALS has resolution to at least $2000 \mathrm{~K}$. 

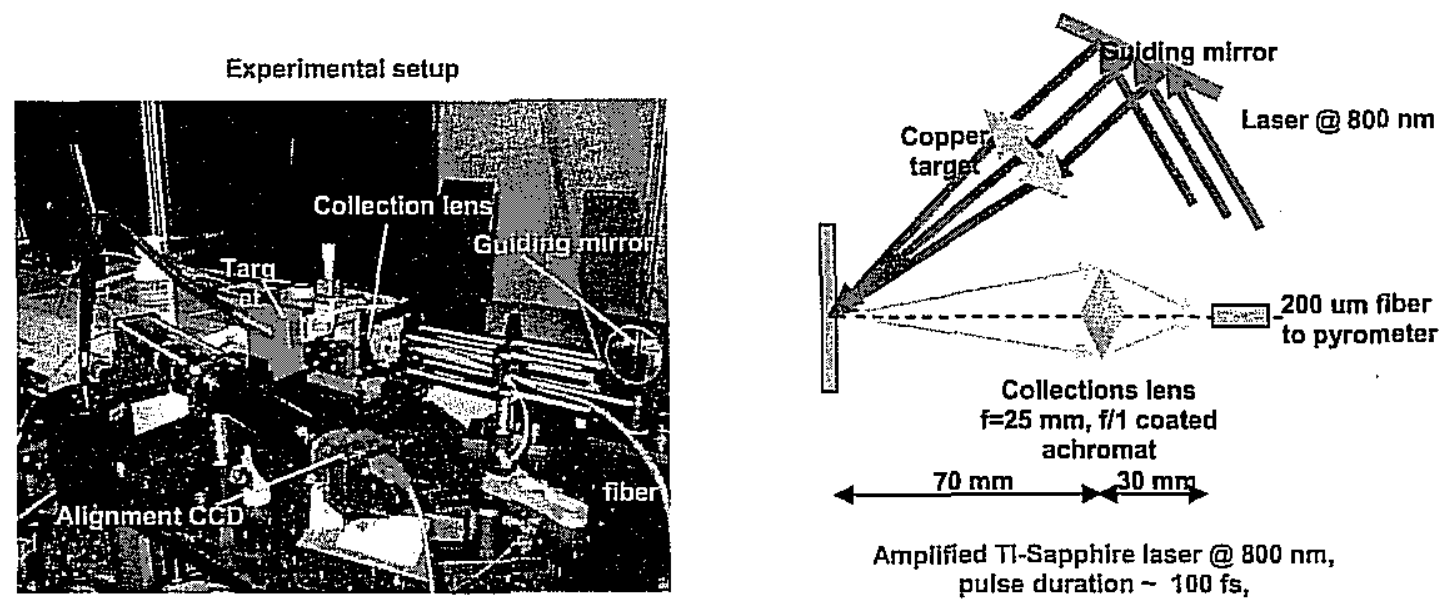
Amplified Tl-Sapphire laser @ $800 \mathrm{~nm}$ pulse duration - $100 \mathrm{fs}$, energy $5-10 \mathrm{~mJ}$, spot size $400 \mathrm{um}$

Copper target: $120 \mathrm{~nm}$ thick $\div 5 \mathrm{~nm}$ of protective carbon on both sldes
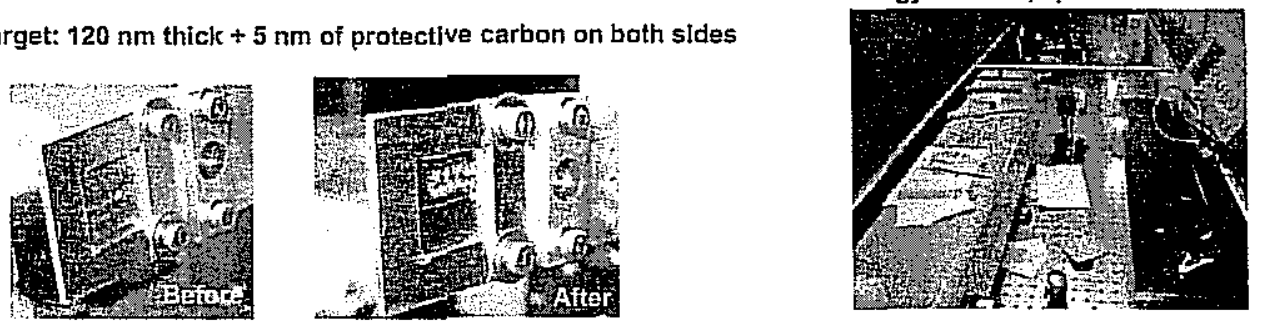

Figure 14. Summary of ALS experimental setup. Clockwise from upper left: assembly of the experiment, schematic of the optical path of the Ti-sapphire heating laser and the pyrometer optics, photographs of the copper target before and after an experiment, and a photograph of the Ti-sapphire laser.

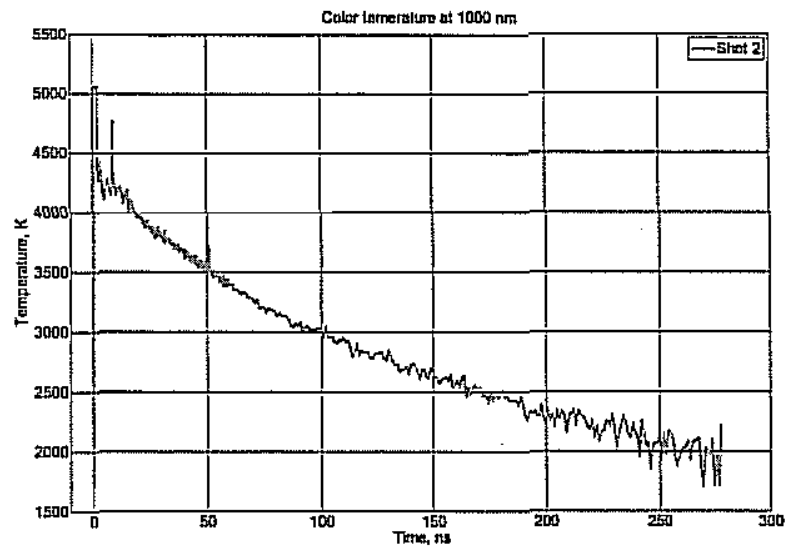

Figure 15. Typical temperature of the copper target in the ALS experiments, as measured by the optical pyrometer, showing the initial spike in target temperature to over $4000 \mathrm{~K}$ and the gradual cooling of the target over a time scale of several hundred nanoseconds. 


\subsection{Streak camera/spectrometer}

Our new Hamamatsu streak camera was bench tested using a pulsed LED. Figure 16 shows some of the details of this test.
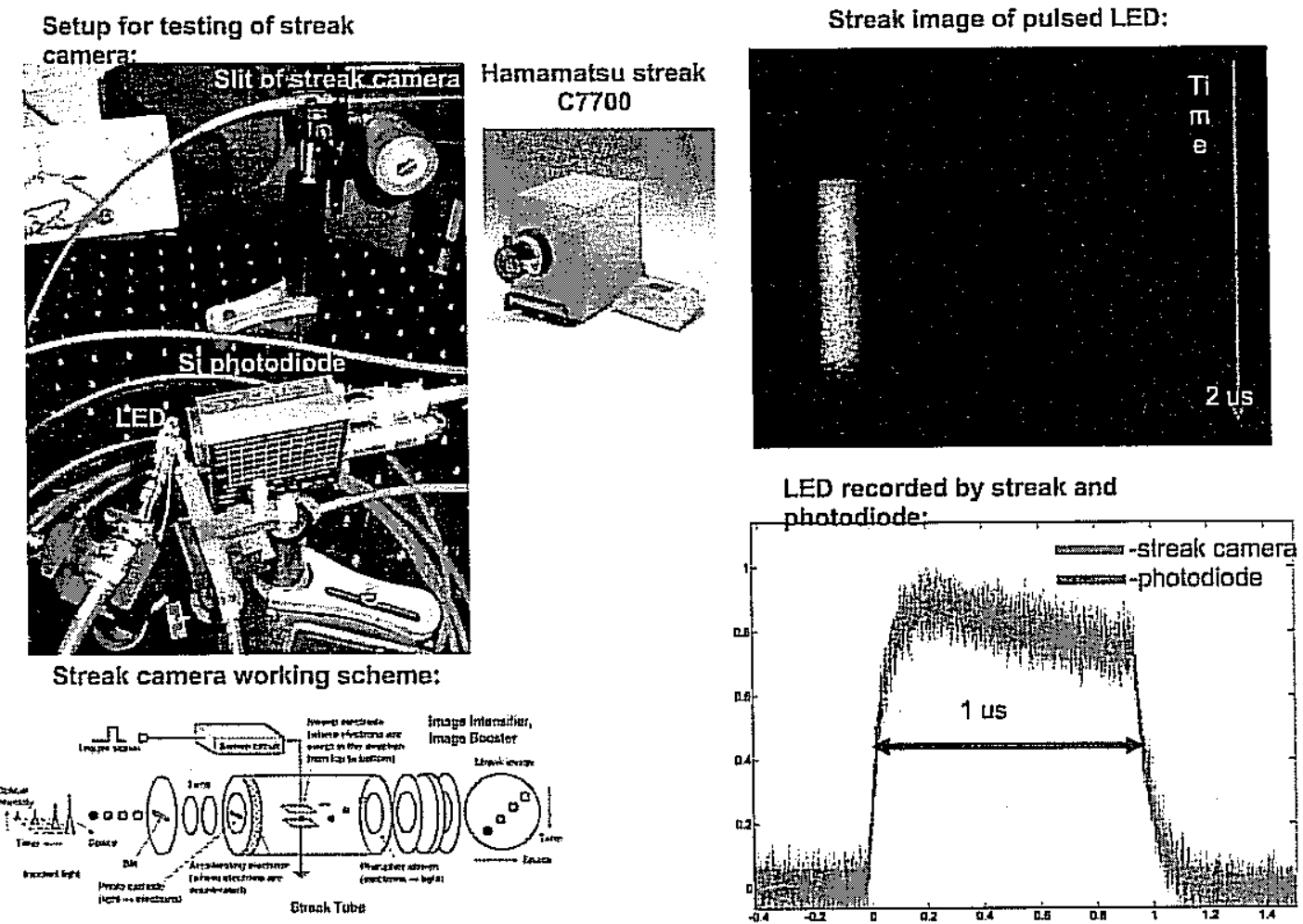

Figure 16. Photographs and signals during acceptance testing of the Hamamatsu streak camera.

In addition, we used the camera in conjunction with a Horiba Jobin Yvon spectrometer grating (PAC CP140-104 CP140 - 104 Spectral range : 250 - $850 \mathrm{~nm}$ over $24.7 \mathrm{~mm}, 285$ grooves $/ \mathrm{mm}$ ) for time resolved spectroscopy of the neutralization plasma on NDCX. Fig. 17 shows sample experimental results from this measurement and Fig. 18 shows a photograph of the streakspectrometer combination. 


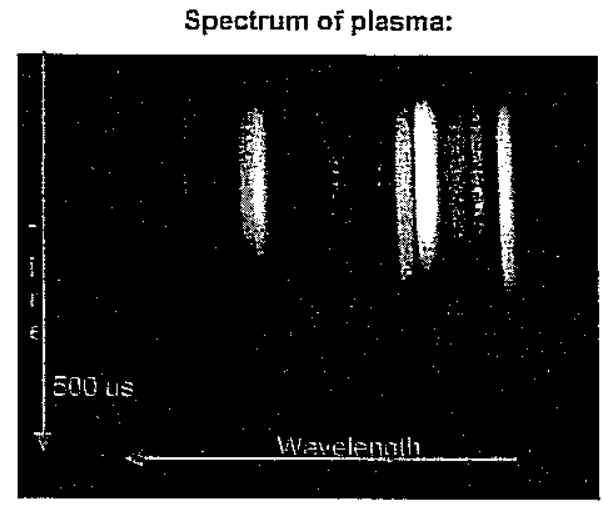

Spectrum of plasma with N2:

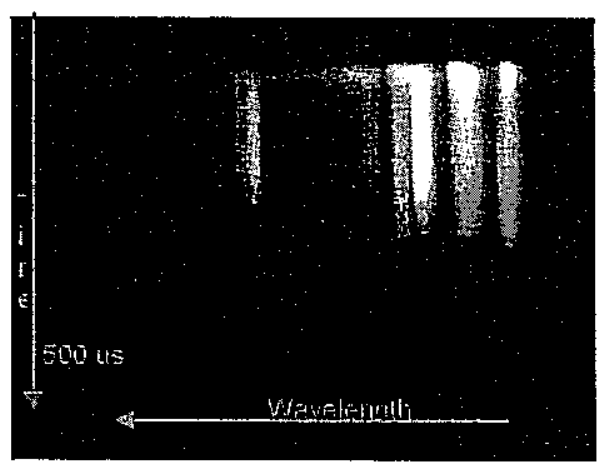

Spectrally resolved:
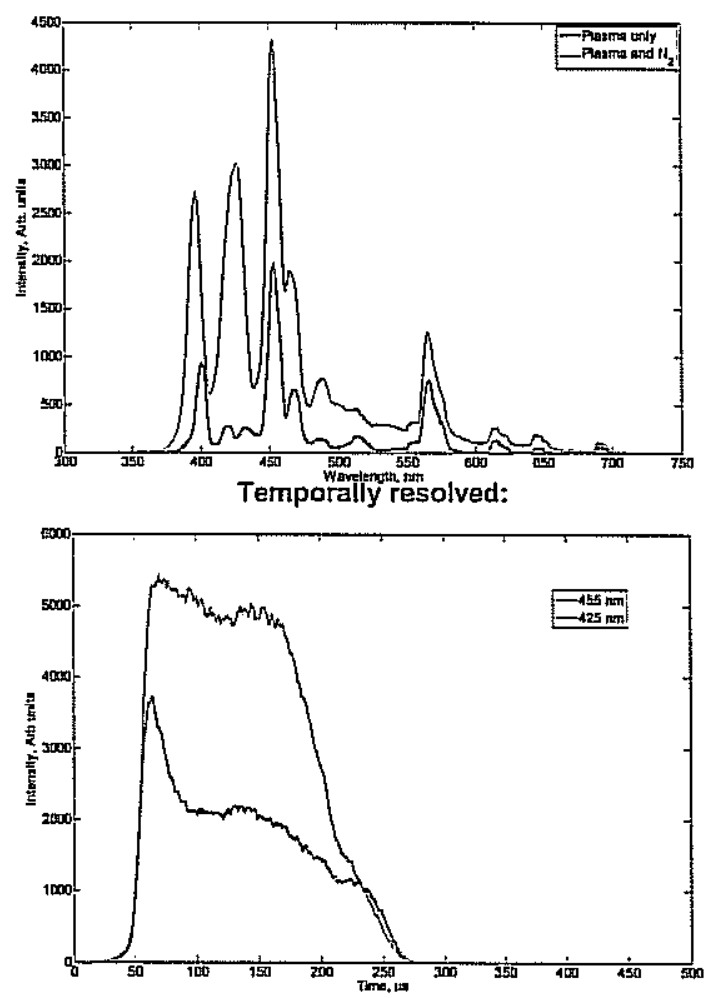

Figure 17. Spectral emission as a function of time and wavelength during a pulse of the aluminum FCAPS plasma in the NDCX target chamber as measured by the streak spectrometer.

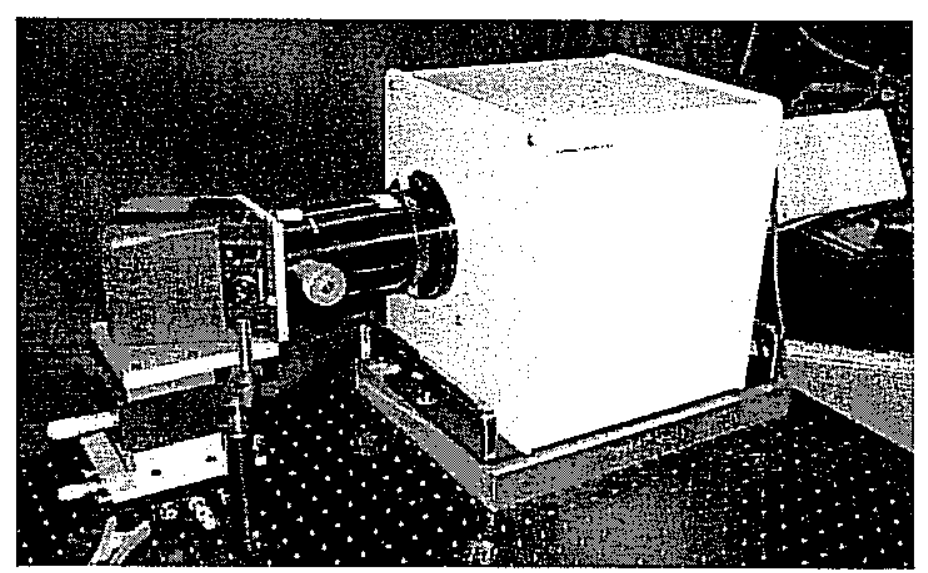

Figure 18. Streak-spectrometer combination as used on NDCX. 


\subsection{VISAR}

We have tested our fiber-coupled VISAR (velocity interferometer system for any reflector, Martin Froescher and Associates) using a conventional roofing nail gun purchased at Home Depot (Fig. 19). A polished piece of aluminum was glued to the piston tip and the probing laser $(1500 \mathrm{~nm}, 15 \mathrm{~mW})$ spot was focused at it using a supplied $50 \mathrm{~mm}$ lens. We used $11 \mathrm{~m}$ meter delay leg, which provides $50 \mathrm{~ns}$ temporal resolution $0.02 \mathrm{~m} / \mathrm{s}$ velocity resolution. In real experiments, we are going to use a $200 \mathrm{~mm}$ delay leg, which will measure velocity with $1 \mathrm{~ns}$ temporal resolution and $4 \mathrm{~m} / \mathrm{s}$ velocity resolution. Also a MATLAB code has been written for velocity extraction from the four curves produced by the instrument. We now know how to align, tune the quadrate, operate and process the data of the VISAR. It is unlikely that the VISAR will be used in the first round of WDM experiment, due to low temperature, i.e. the expansion velocity is expected to be relatively small.
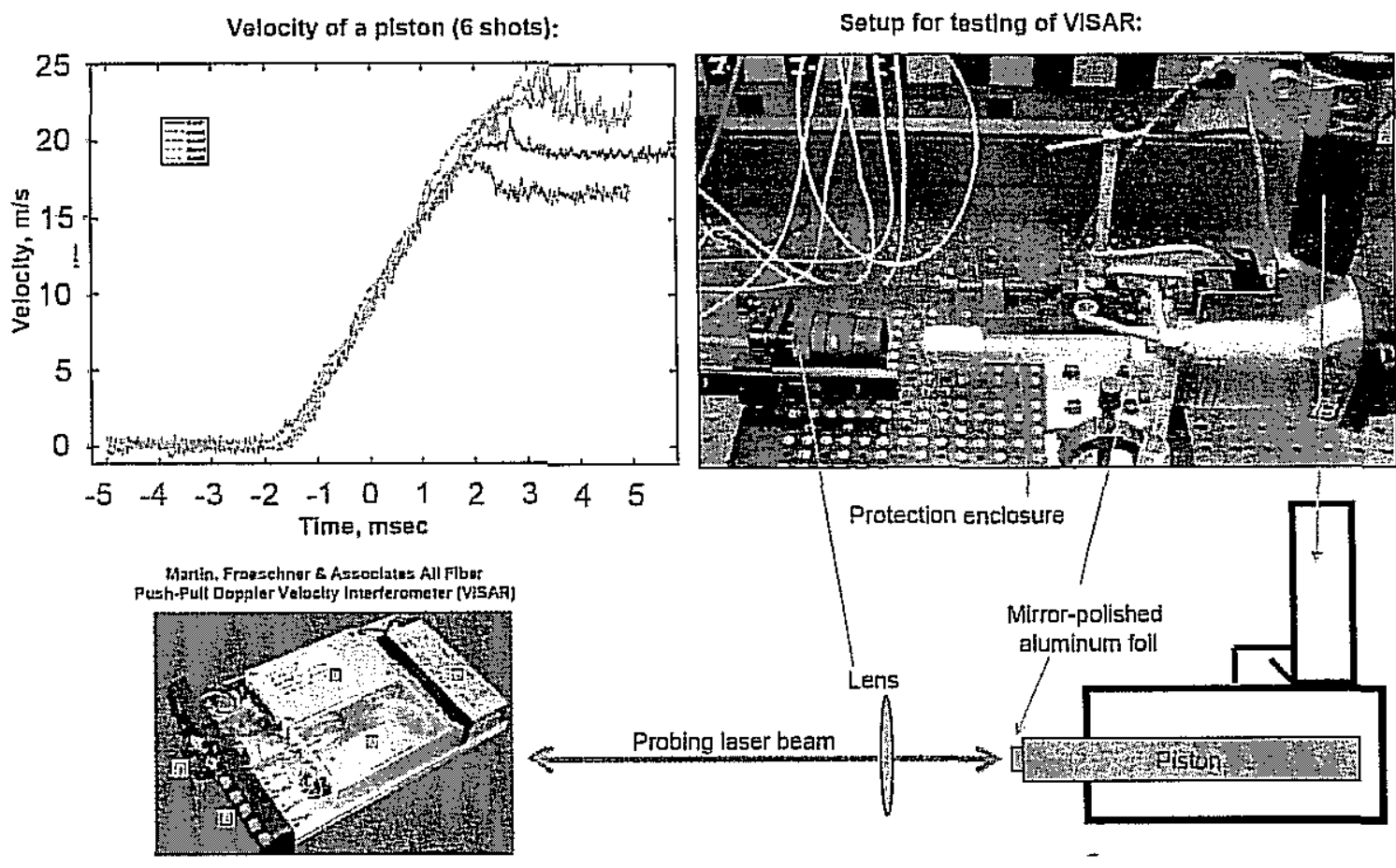

Figure 19. Summary of testing the fiber-coupled VISAR system. Shown are typical test data, a photograph of the test setup, and a schematic sketch of the test setup. 


\section{INSTALLATION ON NDCX}

Fig. 20 shows the installation of the target chamber on NDCX. The target chamber facility is mounted on linear rails to be able to move it in and out of the beamline for servicing and experimental re-arrangements. In support of the target chamber facility we have installed a separate laser room facility in the floor below the target chamber. The laser room can be used for optical target diagnostics pre-alignment and tests. The whole setup including the laser room will be a core capability for any future WDM user facility in the HIFS-VNL.
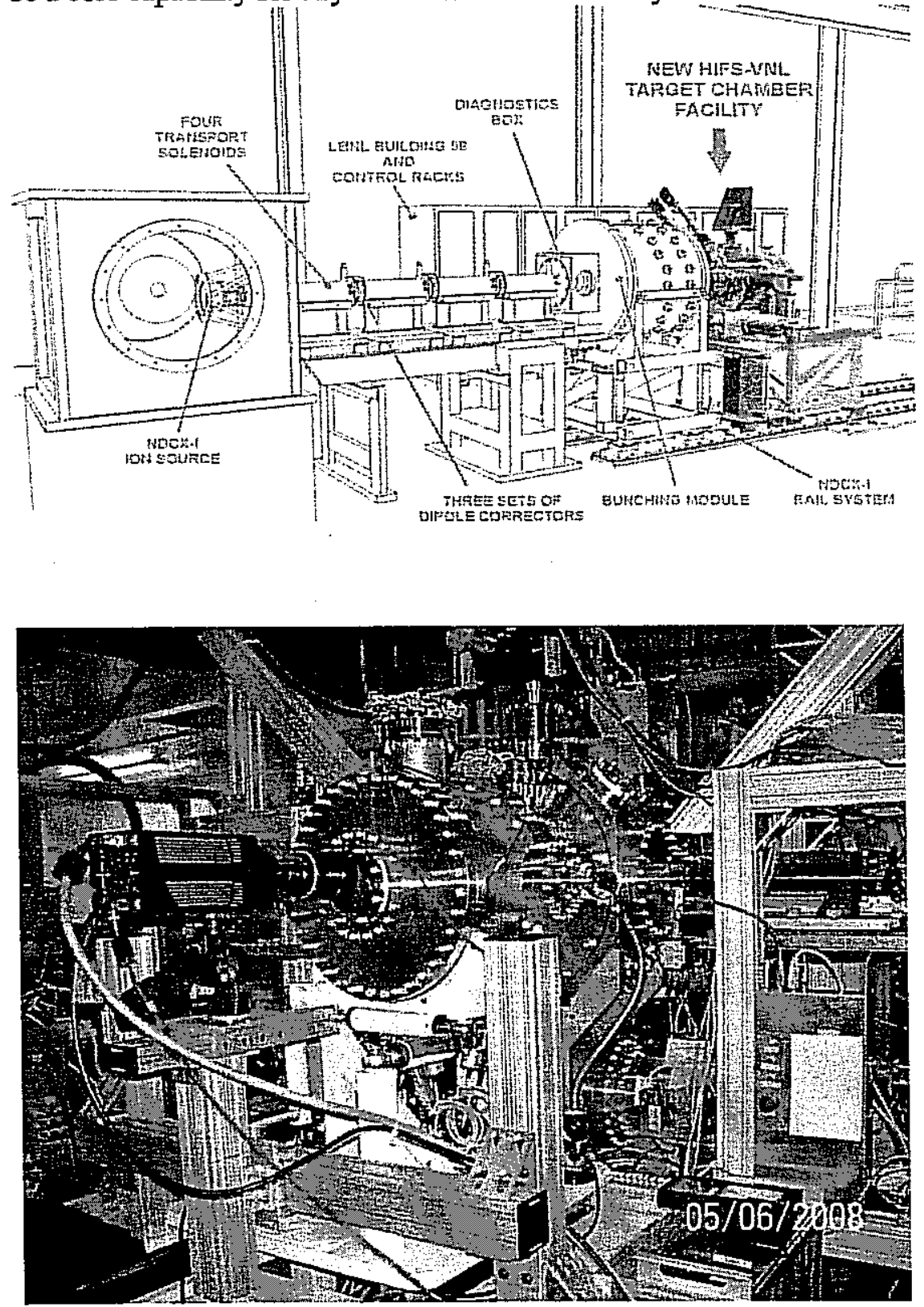

Figure 20: (a) CAD view and (b) photograph of the new HUFS-VNL target chamber facility installed on NDCX-I. 


\section{SUMMARY AND POSSIBLE FUTURE IMPROVEMENTS}

We have described the main components of a novel target chamber facility now available for target experiments on NDCX-I. The same target chamber facility can also be used on future experiments, e.g. NDCX-II, which could provide significantly more beam power.

Several improvements to the target chamber design are conceivable. We will focus on the following three upgrades as soon as possible:

1. Especially for a user facility a robotic target handling system will be required in order to be able to use up to 50 targets without interrupting the experimental program. Such an upgrade will require a vacuum load lock system and a custom designed, vacuum-rated robotic target handling system.

2. Experimental feedback will allow us to refine the design of the optical target diagnostics setup. We plan to move most optical components into the vacuum vessel as close to the target as possible, which will eventually require a new and larger target chamber vacuum vessel.

3. The optical target diagnostics components are currently mounted on manually adjustable $\mathrm{x}-\mathrm{y}-\mathrm{z}$ translation stages. We plan to move most alignment stages into vacuum as close to the target as possible. This will require multiple, vacuum-rated, motorized translation stages.

We will start implementing these improvements once we have gained sufficient experimental experience with the current setup. As future plans for target experiments will evolve we will improve and continually update the target chamber facility to advance the challenging experiments with ion-beam driven, isochorically heated warm dense matter.

\section{REFERENCES}

1. J.J. Barnard, et al., Accelerator and ion beam tradeoffs for studies of warm dense matter, Proc. 2005 Particle Accelerator Conference, p. 2568

2. P.K. Roy, at al., Neutralized drift compression experiments with a high intensity ion beam, NIM A 577 (2007) 223-230.

3. P.A. Seidl, et al., Plans for longitudinal and transverse neutralized beam compression experiments, and initial results from solenoid transport experiments, NIM A 577 (2007) 215-222.

4. F.M. Bieniosek, J.J. Barnard, M.A. Leitner, R.M. More, P.K. Roy, Diagnostics for nearterm warm dense matter experiments, NIM A 577 (2007) 284-288.

5. HIFS-VNL $4^{\text {th }}$ Quarter 2007 milestone report 